\title{
D05 Integrated Reservoir Characterisation in a Carbonate Reservoir
} Kuifu Du, Shell

In this large carbonate platform reservoir, there are two distinct regions identifiable from seismic data: platform interior and raised margin. Whilst all available datasets such as well tests, seismic, logs and cores obtained from a large of number of wells drilled to date indicate that the platform interior area has very low permeability with lack of opened natural fractures prior to acid-stimulation jobs (small fractures only seen after the acidstimulation). However massive natural fracture networks of varying size are present across the platform margin area including a large number of geobodies (karsts and sinkholes up to a few hundreds metres wide) that can be identified from the 3D seismic data. The well tests from the platform margin exhibit the homogeneous radial flow with very high total permeability. This talk presents the improved reservoir description by integration of well tests, seismic and other datasets. Analytic and numeric simulation models are used to explain the pressure data observed from the well tests in this complicated naturally fractured reservoir. 